\title{
Integrating PCR-free amplification and synergistic sensing for ultrasensitive and rapid CRISPR/Cas12a-based SARS-CoV-2 antigen detection
}

\author{
Xiangxiang Zhao ${ }^{\mathrm{a}}$, Zhengduo Wang ${ }^{\mathrm{a}}$, Bowen Yang ${ }^{\mathrm{a}}$, Zilong $\mathrm{Li}^{\mathrm{b}}{ }^{\mathrm{b}}$, Yaojun Tong ${ }^{\mathrm{c}}$, Yuhai Bi ${ }^{\mathrm{d}}$, \\ Zhenghong Li $^{\mathrm{b}}$, Xuekui Xia ${ }^{\mathrm{e}}$, Xiangyin Chen ${ }^{\mathrm{a}}$, Lixin Zhang ${ }^{\mathrm{a}}$, Weishan Wang ${ }^{\mathrm{b}}$, Gao-Yi Tan ${ }^{\mathrm{a}, *}$ \\ a State Key Laboratory of Bioreactor Engineering, and School of Biotechnology, East China University of Science and Technology (ECUST), Shanghai, 200237, China \\ ${ }^{\mathrm{b}}$ State Key Laboratory of Microbial Resources, Institute of Microbiology, Chinese Academy of Sciences, Beijing, 100101, China \\ ${ }^{\mathrm{c}}$ State Key Laboratory of Microbial Metabolism, and School of Life Sciences \& Biotechnology, Shanghai Jiao Tong University, Shanghai, 200240, China \\ ${ }^{\mathrm{d}}$ CAS Key Laboratory of Pathogenic Microbiology and Immunology, Institute of Microbiology, Center for Influenza Research and Early-warning (CASCIRE), CAS-TWAS \\ Center of Excellence for Emerging Infectious Diseases (CEEID), Chinese Academy of Sciences, Beijing, 100101, China \\ ${ }^{\mathrm{e}}$ Key Biosensor Laboratory of Shandong Province, Biology Institute, Qilu University of Technology (Shandong Academy of Sciences), Jinan, 250013, China
}

\section{A R T I C L E I N F O}

\section{Keywords:}

CRISPR/Cas12a

Aptamer

Antigen detection

SARS-CoV-2

PCR-Free amplification

Synergistic sensing

\begin{abstract}
A B S T R A C T
Antigen detection provides particularly valuable information for medical diagnoses; however, the current detection methods are less sensitive and accurate than nucleic acid analysis. The combination of CRISPR/Cas12a and aptamers provides a new detection paradigm, but sensitive sensing and stable amplification in antigen detection remain challenging. Here, we present a PCR-free multiple trigger dsDNA tandem-based signal amplification strategy and a de novo designed dual aptamer synergistic sensing strategy. Integration of these two strategies endowed the CRISPR/Cas12a and aptamer-based method with ultra-sensitive, fast, and stable antigen detection. In a demonstration of this method, the limit of detection was at the single virus level $(0.17 \mathrm{fM}$, approximately two copies $/ \mu \mathrm{L}$ ) in SARS-CoV-2 antigen nucleocapsid protein analysis of saliva or serum samples. The entire procedure required only $20 \mathrm{~min}$. Given our system's simplicity and modular setup, we believe that it could be adapted reasonably easily for general applications in CRISPR/Cas12a-aptamer-based detection.
\end{abstract}

\section{Introduction}

Antigens have been used as diagnostic hallmarks for many diseases, particularly infection with viruses, for example, severe acute respiratory syndrome coronavirus 2 (SARS-CoV-2), the cause of the current global coronavirus disease 2019 (COVID-19) pandemic [1]. Many methods have been developed for SARS-CoV-2 detection through nucleic acids, antigens, and antibodies. Although PCR-based nucleic acid detection is the most accepted and reliable approach, it is relatively slow and device-dependent, owing to the requirements of RNA extraction and reverse transcription. In addition, RNA is less stable than protein (antigen) in laboratory conditions [2,3]. Moreover, antibody-based tests work well only in late stages of infection, after patients have mounted immune responses, and therefore are not suitable for early diagnosis [4-6]. In comparison, antigen-based SARS-CoV-2 detection has several unique merits in COVID-19 diagnosis. Given its robustness and simplicity, it provides an important addition to the toolbox for
SARS-CoV-2 detection [3,7-9]. However, compared with mature nucleic acid detection approaches, recent state-of-the-art methods for SARS-CoV-2 antigen detection have some deficiencies in low abundance viral sample detection (e.g., early diagnosis) [3]. The underlying reason is that signal amplification of low amounts of antigen is not possible. In contrast, the amount of antibodies are already amplified in vivo after antigen stimulation, and nucleic acids can be amplified in vitro by PCR or LAMP (loop-mediated isothermal amplification) [10], thus boosting both signals. To address the challenge of signal amplification for antigen detection, we endeavor to develop a novel antigen detection system with high sensitivity and excellent performance by amplifying the sensing signals.

CRISPR/Cas-based nucleic acid detection systems, such as SARSCoV-2 DETECTR [11], SHEROLOCK [12], STOPCovid [13], and AIOD-CRISPR [14], have been developed and applied in rapid diagnostic tests for SARS-CoV-2. The advantages of CRISPR/Cas-based detection systems have been well demonstrated, and these methods

\footnotetext{
Peer review under responsibility of KeAi Communications Co., Ltd.

* Corresponding author.

E-mail address: tangy@ecust.edu.cn (G.-Y. Tan).
} 
have shown great potential during the COVID-19 pandemic, owing to their superior speed, portability, low cost, and comparable sensitivity to that of the traditional gold standard of RT-qPCR assays (one copy/ $\mu \mathrm{L}$ of viral RNA) [15-18]. However, the CRISPR/Cas-based detection paradigm has not been expanded to SARS-CoV-2 antigen detection [19]. To fill this gap, we proposed that the abovementioned antigen detection challenges could be overcome by coupling CRISPR/Cas and a de novo designed highly efficient and stable signal amplification system.

Aptamers as a general biosensing platform can be applied to biosensing or detection of a broad range of analytes [20,21]. Previously, CRISPR/Cas12a and aptamer-mediated systems for detection of diverse analytes (e.g., proteins or small molecules) have been developed [15, 22]. These platforms directly translate the signal of aptamer responsive target molecules into a CRISPR-mediated nucleic acid detection-based output signal. However, owing to the lack of stable amplification of the input signal, the detection sensitivity this method has been unable to meet the requirements for low abundance sample detection or early diagnosis of COVID-19, in which the viral titer is usually as low as several copies per microliter (e.g., in saliva) [23].

Here, to improve the sensitivity or sensing efficiency of the CRISPR/ Cas12a and aptamer-mediated method and achieve ultrasensitive detection, we innovated two aspects for the configuration of this new antigen detection platform. First, we developed a PCR-free amplification strategy to convert the aptamer-antigen recognition signal to more trigger dsDNA signal. Second, we undertook de novo design of a dual aptamer synergetic module to more sensitively sense the antigen independently of the affinity between the aptamer and antigen. In a demonstration of this platform, we successfully identified two copies $/ \mu \mathrm{L}$ SARS-CoV-2 in samples by using our newly established system of antigen detection; the LOD was comparable to that of the gold standard of PCRbased nucleic acid detection, but the detection was more rapid $(\sim 20$ $\min$ ) and the costs were lower.

\section{Materials and methods}

\subsection{Materials and reagents}

Aptamers and primers were chemically synthesized by Beijing Tsingke Biotech Co. Ltd (Beijing, China) (Table S1). Beaver Beads ${ }^{\mathrm{TM}}$ streptavidin (1 $\mu \mathrm{m}$ average diameter) was purchased from Beaver (Suzhou, China). SARS-CoV-2 Np was purchased from Sino Biological Inc (Beijing, China). Human serum and other chemicals for buffers and solvents were purchased from Sigma-Aldrich, Inc. (Shanghai, China). UltraPure DNase/RNase-free Distilled Water, HiScribe T7 Quick High Yield RNA Synthesis Kit, Taq $2 \times$ Master Mix, standard Taq buffer and RNase inhibitor were purchased from New England BioLabs Inc. (Ipswich, UK). The TIANquick Maxi Purification Kit was purchased from Tiangen Biotech Co. Ltd (Beijing, China). The RNA Clean \& Concentrator ${ }^{\mathrm{TM}}-5$ was purchased from Zymo Research (Irvine, CA). All chemicals and reagents used were of analytical grade and were prepared with deionized water $\left(18 \mathrm{M} \Omega \mathrm{cm}\right.$ ) from a Milli-Q ${ }^{\circledR}$ Water Purification System (Millipore, Billerica, MA, USA). The portable fluorometer was purchased from Thermo Fisher Scientific Inc.

\subsection{Cas12a protein expression and purification}

Cas12a (LbCas12a) from Lachnospiraceae bacterium ND2006 was expressed and purified as described in our previous study. Briefly, Escherichia coli BL21(DE3) was transformed with the pET28TEVLbCas12a expression plasmid and grown overnight in Luria-Bertani medium at $37{ }^{\circ} \mathrm{C}$ with shaking at $150 \mathrm{rpm}$ until the exponential growth phase. Protein expression was then induced with $100 \mu \mathrm{M}$ isopropyl- $\beta$-D-thiogalactoside, and the cells were cultured at $16^{\circ} \mathrm{C}$ for $12 \mathrm{~h}$. The cells were harvested by centrifugation at $5000 \times g$. For protein purification, the cell pellets were resuspended in lysis buffer $(50 \mathrm{mM}$ Tris$\mathrm{HCl}$ [pH 7.4], $200 \mathrm{mM} \mathrm{NaCl}, 2 \mathrm{mM}$ dithiothreitol, and 5\% glycerol) supplemented with EDTA-free protease inhibitors (Sigma-Aldrich, Roche Diagnostics), and then lysed with ultrasonication. The lysate was loaded onto a HisTrap FF column (GE Healthcare) and washed with an imidazole concentration gradient. The peak fractions were collected and desalted with dialysis. The solution was then loaded onto a HiTrap Q HP column (GE Healthcare), and the peak fractions were collected, pooled, and concentrated. The concentrated solution was loaded onto a HiLoad $16 / 600$ Superdex $200 \mathrm{pg}$ column for fast protein liquid chromatography (AKTA Explorer 100; GE Healthcare). The gel filtration fractions were analyzed with SDS-PAGE, and the protein concentrations were determined with the Bradford method. The purified product was dissolved in storage buffer (20 mM Tris- $\mathrm{HCl}$ [pH 7.5], $1 \mathrm{M} \mathrm{NaCl}$, and $50 \%$ glycerol) and stored at $-80^{\circ} \mathrm{C}$ until use.

\subsection{HyDNA, crRNA, and Y-DNA preparation}

The oligonucleotides used in this work are listed in Table S1. To prepare the template for HyDNA amplification, a DNA sequence with ten copies of Cas12a trigger dsDNA was synthesized. Then, C3 modified forward primers (com1F, com2F, com3F, com4F, com5F, com6F, com7F, com8F, com9F, com10F, com11F, com12F, com13F, com14F, com15F, com16F, com17F, com18F, com19F, and com20F) combined with reverse primers (dsDNA-R1, dsDNA-R2, dsDNA-R3, dsDNA-R4, dsDNA-R5, dsDNA-R6, dsDNA-R7, dsDNA-R8, dsDNA-R9, and dsDNAR10) were amplified by using the synthesized template DNA to produce the HyDNAs containing Cas12a trigger dsDNA and different comssDNA sequences (ssDNA complementary to part of the sequence of the aptamer) [24]. The PCR products were generated with Taq $2 \times$ Master Mix. The PCR conditions were $1 \mathrm{~min}$ at $95{ }^{\circ} \mathrm{C}$ for activation followed by 30 cycles at $95^{\circ} \mathrm{C}$ for $30 \mathrm{~s}$ for denaturation, $55^{\circ} \mathrm{C}$ for $30 \mathrm{~s}$ for annealing, $68^{\circ} \mathrm{C}$ for $30 \mathrm{~s}$ for elongation, and a final cycle at $68^{\circ} \mathrm{C}$ for 10 min for final elongation. The HyDNAs were purified with a TIANquick Maxi Purification Kit.

To prepare the template for crRNA (crRNA1, crRNA2, crRNA3, crRNA4, crRNA5, crRNA6, crRNA7, crRNA8, crRNA9, and crRNA10) synthesis, paired oligonucleotides containing a T7 priming site (Table S1) were synthesized and annealed in $1 \times$ Taq DNA Polymerase PCR Buffer. The crRNAs were then transcribed with a HiScribe ${ }^{\mathrm{TM}} \mathrm{T} 7$ Quick High Yield RNA Synthesis Kit and purified with RNA Clean \& Concentrator $^{\mathrm{TM}}-5$.

To obtain the Y-DNA, three oligonucleotides (Y1, Y2, and Y3) were designed. Each oligonucleotide sequence was complementary to the other two oligonucleotide sequencess. For the preparation of the Y-DNA based biosensor, the produced Y-DNA contained 25 bases as a sticky end on the two arms ( $\mathrm{Y} 1$ and $\mathrm{Y} 2$ ) and a biotin labeled poly A end on the Y3 arm. For construction of Y-DNA, the same molar amounts of Y1, Y2, and Y3 were mixed with Taq DNA Polymerase PCR buffer. Then the mixture was incubated at $95{ }^{\circ} \mathrm{C}$ for $2 \mathrm{~min}$, quickly cooled to $65^{\circ} \mathrm{C}$, incubated at $65^{\circ} \mathrm{C}$ for $2 \mathrm{~min}$, cooled to $60^{\circ} \mathrm{C}$ at a rate of $-1{ }^{\circ} \mathrm{C}$ per min, incubated at $60{ }^{\circ} \mathrm{C}$ for $2 \mathrm{~min}$, cooled to $20^{\circ} \mathrm{C}$ at a rate of $-0.1^{\circ} \mathrm{C}$ per $30 \mathrm{~s}$, and finally cooled to $16^{\circ} \mathrm{C}$ [25]. The resulting HyDNAs and crRNAs were quantified with a NanoDrop 2000 spectrophotometer (Thermo Fisher Scientific). The HyDNAs and Y-DNA were stored at $4{ }^{\circ} \mathrm{C}$. The crRNAs were stored at $-80{ }^{\circ} \mathrm{C}$. Throughout all experiments, RNase-free materials and conditions were used.

\subsection{Biosensor configuration and sensing}

To construct the one aptamer-based biosensor, $\mathrm{MB}$, biotin labeled aptamer, and HyDNA were used. First, $20 \mu \mathrm{L}$ of $10 \mathrm{mg} / \mathrm{mL} \mathrm{MB}$ was washed with $1 \times$ PBS containing Tween 20 (PBST) buffer to remove the residual $\mathrm{NaN}_{3}$ protection solution. Then $30 \mu \mathrm{L}$ of $10 \mu \mathrm{M}$ biotin labeled aptamer in $1 \times$ PBST buffer was added to the MB solution and mixed well for $30 \mathrm{~min}$ at room temperature. The aptamer-coated MB were then washed twice with buffer to remove excess biotin labeled aptamer. Then the HyDNA $(0.5 \mu \mathrm{M}, 20 \mu \mathrm{L})$ was added to the solution and mixed well at 
room temperature for $20 \mathrm{~min}$. After the sample was washed three times with buffers to remove excess HyDNAs, the MB-aptamer-HyDNA complex was dispersed in $1 \mathrm{~mL}$ of $1 \times$ PBST buffer. The MB in each $20 \mu \mathrm{L}$ aliquot of this solution were used in this work.

To construct the dual aptamer-based biosensor, MB, biotin labeled YDNA, adapter1-A48, adapter2-A61, com10-HyDNA and com11-HyDNA were used. First, $20 \mu \mathrm{L}$ of $10 \mathrm{mg} / \mathrm{mL}$ MB was washed with $1 \times$ PBST buffer to remove the residual $\mathrm{NaN}_{3}$ protection solution. Then $300 \mu \mathrm{L}$ of $1 \mu \mathrm{M}$ biotin labeled Y-DNA was added to the MB solution and mixed well for $30 \mathrm{~min}$ at room temperature. The MB were then washed twice with buffer to remove excess Y-DNA. The adapter1-A48 $(10 \mu \mathrm{M}, 30 \mu \mathrm{L})$ and adapter2-A61 (10 $\mu \mathrm{M}, 30 \mu \mathrm{L})$ were added to the solution and mixed well at room temperature for $20 \mathrm{~min}$. The $\mathrm{MB}$ were washed twice with buffer to remove excess aptamers. The HyDNAs $(0.5 \mu \mathrm{M}, 10 \mu \mathrm{L})$ were added to the solution and mixed well at room temperature for $20 \mathrm{~min}$. After the sample was washed three times with buffers to remove excess HyDNAs, the MB-YDNA-aptamer-HyDNA complex was dispersed in $1 \mathrm{~mL}$ of $1 \times$ PBST buffer. The MB contained in each $20 \mu \mathrm{L}$ aliquot of this solution were used in this work.

To assess the feasibility of the platform, we incubated samples with different concentrations of $\mathrm{Np}$ with the biosensor at room temperature for $20 \mathrm{~min}$. Each solution was then separated with a magnetic rack, and $10 \mu \mathrm{L}$ of supernatant was transferred into a new centrifuge tube for quantification with the Cas12a-based FQ-labeled reporter system.

\subsection{Quantification of HyDNA with the CRISPR-Cas12a reporting system}

In the CRISPR-Cas12a cutting system, NEB CutSmart ${ }^{\circledR}$ Buffer was used as the reaction buffer. For the collateral cleavage assay, an equimolar ratio of Cas12a and crRNA was premixed with the FQ-labeled reporter in NEB CutSmart ${ }^{\circledR}$ Buffer and then distributed in a 384-well plate for the subsequent experiment (Table S2). The diluted supernatant containing the HyDNA was added to the reporter system, and the fluorescence signal was detected every $1.5 \mathrm{~min}$ with an EnSpire ${ }^{\mathrm{TM}}$ Multimode Plate Reader (PerkinElmer, Inc., USA) at an excitation wavelength of $480 \mathrm{~nm}$ and an emission wavelength of $520 \mathrm{~nm}$.

\subsection{Analysis of samples}

An inactivated SARS-CoV-2 sample (hCoV-19/China/CAS-B001/ 2020, National Microbiology Data Center NMDCN0000102-3, GISAID databases EPI_ISL_514256-7) was obtained from Dr. Bi and was quantified previously by using gene $\mathrm{N}$ primers (Fig. S12). To demonstrate the analytical reliability and practical application of the biosensing platform, we tested swab samples spiked with different concentrations of inactivated SARS-CoV-2. First, $10 \mu \mathrm{L}$ inactivated SARS-CoV-2 sample was incubated in $10 \mu \mathrm{L}$ lysis buffer for $10 \mathrm{~min}$ and serially diluted in half to obtain different concentrations of lysed samples. Each diluted sample ( $2 \mu \mathrm{L}$ ) was added to $18 \mu \mathrm{L}$ of the biosensor system and incubated for 20 min. To construct the calibration curve, we detected the HyDNA in the supernatant with the Cas12a-based FQ-labeled reporter system. The changes in the fluorescence intensity over time were measured, and the slope of the fluorescence curve in the linear region between 0 and 25 min (normally) represented the ssDNA trans-cleavage rate of CRISP$\mathrm{R}-\mathrm{Cas12a}$. The linear relationship between the trans-cleavage rate (or slope) and the SARS-CoV-2 standard concentration was obtained.

To demonstrate that SARS-CoV-2 screening with our platform would be possible outside of laboratory settings, we used a portable fluorometer (Qubit ${ }^{\mathrm{TM}} 4$ Fluorometer) to quantify the fluorescence signal generated by the detection assay. The unactivated SARS-CoV-2 sample was diluted to three to five copies/ $\mu \mathrm{L}$, and $\mathrm{Np}$ was released with lysis buffer. The extracted $\mathrm{Np}$ sample $(2 \mu \mathrm{L})$ was added to $20 \mu \mathrm{L}$ of the biosensor system and incubated for $20 \mathrm{~min}$. Then the HyDNA in the supernatant was detected with the Cas12a-based FQ-labeled reporter system. The fluorescence signal increased in $1 \mathrm{~min}$ was detected with the portable fluorometer at an excitation wavelength of $470 \mathrm{~nm}$ and an emission wavelength of $510-580 \mathrm{~nm}$.

\subsection{Data analysis}

All experiments and assays were repeated at least three times. The data in all figures are expressed as means and standard deviation (SD). Microsoft Excel 2016, Origin 8, and GraphPad Prism version 6.0 were used for data analysis.

\section{Results}

\subsection{Design and construction of a prototype CRISPR/Cas12a and} aptamer-mediated platform for SARS-CoV-2 antigen detection

The SARS-CoV-2 viral surface contains four types of proteins, i.e., spike (S), nucleocapsid (N), membrane (M), and envelope (E), of which S protein and $\mathrm{N}$ protein have been used in antigen detection [3,26,27]. In this study, to couple antigen recognition with a CRISPR-based detection system, we used a specific aptamer (A48, with an equilibrium dissociation constant $\left(K_{\mathrm{D}}\right)$ of $\left.0.49 \mathrm{nM}\right)$ [27] of $\mathrm{N}$ protein as the recognition element to develop a SARS-CoV-2 antigen biosensor with our recently established CRISPR/Cas12a-based biosensing platform [15]. Our prototype was denoted the antigen biosensing platform CaT-Smelor-Covid. v1 (CRISPR/Cas12a and aptamer-mediated detector of diverse analytes for COVID-19, version 1). The working principle of CaT-Smelor-Covid. v1 is illustrated in Fig. 1a. In brief, a hybrid DNA (HyDNA) containing the Cas12a triggering dsDNA and a single-stranded DNA (ssDNA) complementary to parts of the aptamer A48 sequence was anchored to A48-coated magnetic beads (MB) (Fig. S1). SARS-CoV-2 nucleocapsid protein (Np), if present, interacts with A48 and releases the HyDNA. The released HyDNA then triggered Cas12a to cleave the fluorophore quencher (FQ)-labeled ssDNA probe, thus producing a readable fluorescence signal output. After optimizing the reaction system and signal-to-noise (S/N) ratio (Figs. S2 and S3), we found that the linear detection range of $\mathrm{Np}$ was $0.19-781 \mathrm{pM}\left(R^{2}>0.99\right)$ (Fig. 1b, c, Fig. S4) with a LOD of $32 \mathrm{fM}$ (approximately 193 copies $/ \mu \mathrm{L}$ ). For nucleic acid detection, the LOD of the gold-standard RT-qPCR and CRISPR-based methods reached up to one copy/ $\mu \mathrm{L}$ of viral RNA [9]. Thus, this prototype did not meet the requirements for detection of SARS-CoV-2 in saliva samples or early diagnosis. Developing novel strategies for sensitive sensing and stable amplification is key for this CRISPR-based antigen detection paradigm.

\subsection{Introduction of a PCR-free strategy to amplify the CRISPR/Cas12a generated signal}

In the CRSIR/Cas12a and FQ-based signal output and display modules, the slopes of the fluorescence signal are proportional to the concentration of the released triggering dsDNA [15]. To increase or amplify the signal generated by the Cas12a triggering dsDNA, we designed a cluster of multiple triggering dsDNAs in tandem. Considering the trade-off between the $\mathrm{S} / \mathrm{N}$ ratio and amplified signal, we kept the length of dsDNA below $600 \mathrm{bp}$, a length accommodating as many as 10 copies of the Cas12a triggering dsDNA with a 30 bp interval sequence (Fig. S5) [28]. Then the HyDNA containing different copy numbers (one to ten) of Cas12a triggering dsDNAs was anchored to A48-coated MB (Fig. S6). The slopes of the fluorescence signal were proportional to one to ten copies of Cas12a triggering dsDNA (HyDNA10; Fig. 2c and d, Fig. S7). Thus, we constructed CaT-Smelor-Covid.v2 (version 2) by using HyDNA10 instead of HyDNA01 in the CaT-Smelor-Covid.v1 (Fig. 2a and b). When detecting $\mathrm{Np}$, we observed that the slope of the fluorescence intensity was linear $\left(R^{2}>0.99\right)$, and the detection range was 6-381 fM (Fig. 2e, f, Fig. S8) with a LOD of $3.48 \mathrm{fM}$ (approximately 42 copies $/ \mu \mathrm{L}$ ), thus indicating that the introduction of clustered multi-copy Cas12a triggering dsDNA indeed boosted the signal. With the introduction of a PCR-free signal amplification procedure, CaT-Smelor-Covid.v2 showed 
a

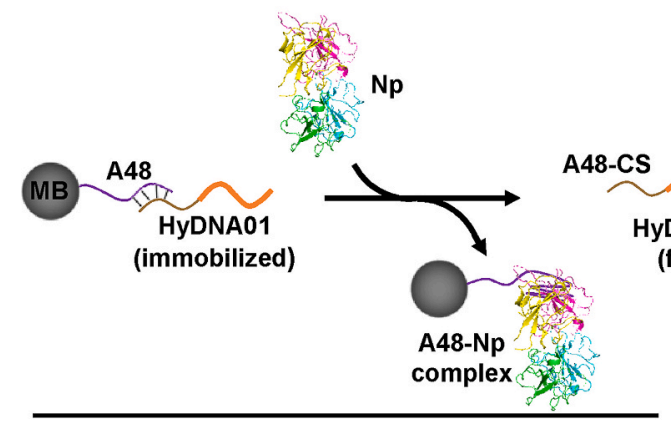

Aptamer-based Biosensing

(Antigen signal $\rightarrow$ Nucleic acid signal)

b

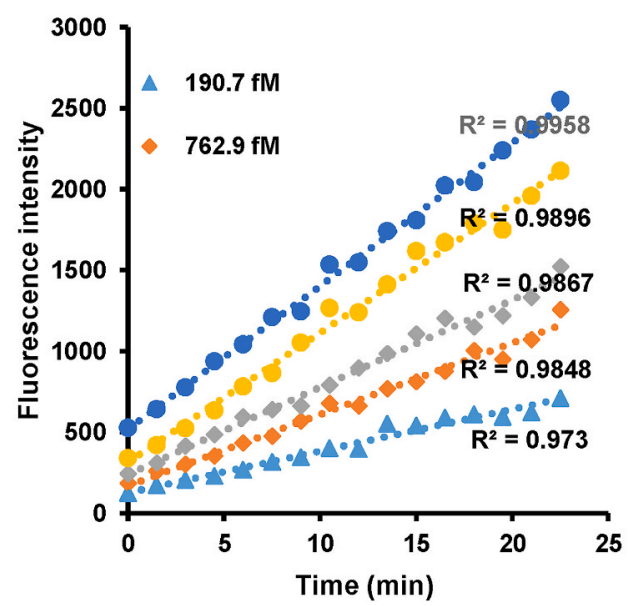

crRNA

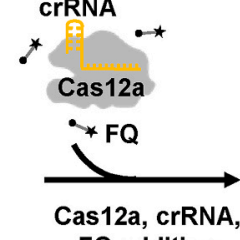

FQ addition

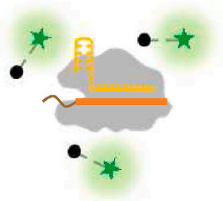

CRISPR/Cas12a-based Detection

(Nucleic acid signal $\rightarrow$ Fluorescence)

C

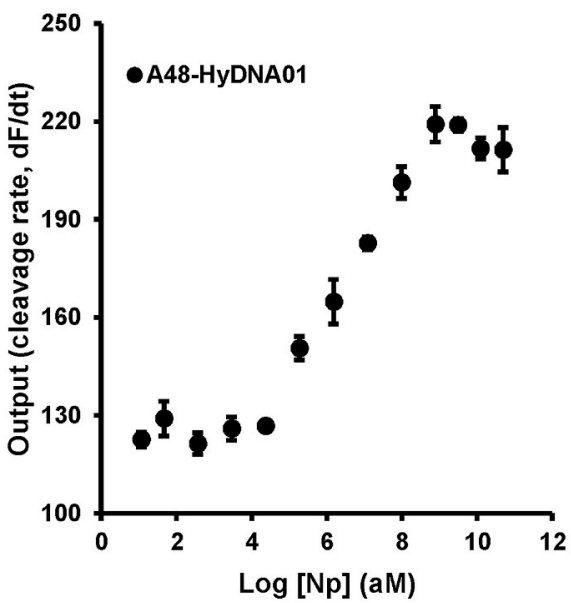

Fig. 1. Detection of Np with the CaT-Smelor-Covid.v1 platform. (a) Schematic diagram of CaT-Smelor-Covid.v1. MB: streptavidin-coated magnetic bead. A48: aptamer targeting the SARS-CoV-2 nucleocapsid protein. HyDNA01: HyDNA containing a single Cas12a trigger dsDNA. Np: nucleocapsid protein. (b) Readout of the fluorescence signal by the CaT-Smelor-Covid.v1 platform in response to Np. The background signal (control) has been subtracted from the values displayed in the graph. (c) Relationship between the slope and Np concentration output with the prototype antigen biosensing platform. A48-HyDNA01: core component of the CaTSmelor-Covid.v1 platform. The data are the means and SD of three independent replicates.

the expected characteristics of high stability and rapid detection. However, the LOD was inadequate for real-world detection in saliva samples or early diagnosis.

\subsection{Introduction of $Y$-shaped DNA-based aptamers to synergistically increase the sensing sensitivity}

To further improve the detection sensitivity of CaT-Smelor-Covid.v2, we pursued a de novo synergistic strategy in CaT-Smelor-Covid.v3 (version 3) by applying two different aptamers recognizing different epitopes of Np to release two cognate tandem HyDNA10 molecules (Fig. 3a and b). When one aptamer binds the antigen and releases HyDNA, it promotes the response of the other aptamer to the same antigen and the release HyDNA due to proximity, thus producing a synergistic effect. Here, we introduced a biotin labeled Y-shaped DNA to simultaneously anchor the aptamer (A48) and another previously characterized aptamer (A61, $K_{\mathrm{D}}=2.74 \mathrm{nM}$ ) [27] on the MB. Then the two optimized HyDNA10 molecules (Fig. S9) were anchored on MB via interaction with aptamers A48 and A61. With CaT-Smelor-Covid.v3, we achieved excellent $\mathrm{Np}$ detection. Trace amounts of $\mathrm{Np}$ at concentrations of 0.19-2.98 fM were unambiguously and readily detected, with an LOD of $0.17 \mathrm{fM}$ (Fig. 3c, d, Fig. S11). The LOD of SARS-CoV-2 was approximately two copies $/ \mu \mathrm{L}$, the lowest value reported to date for SARS-CoV-2 antigen detection through a CRISPR/Cas-based method [3,19]. As expected, the response signal of CaT-Smelor-Covid.v3 allowed us to calculate a Hill coefficient of approximately 1.5 , thus indicating positive cooperativity.

3.4. Rapid and sensitive detection of inactivated SARS-CoV-2 in clinical samples

The LOD of CaT-Smelor-Covid.v3 for antigen detection was very close to that of the gold standard of RT-qPCR and CRISPR/Cas-based nucleic acid detection $[11,13]$, thus indicating that this platform had great potential for real-life applications. We then tested the performance of our CaT-Smelor-Covid.v3 on authentic sample detection by using inactivated SARS-CoV-2 [29]. As shown in Fig. 4a, as few as two copies/ $\mu$ L SARS-CoV-2 could be efficiently detected $(P<0.05)$. Moreover, the entire process required only approximately $20 \mathrm{~min}$ with a commercially available portable fluorescence detector (Qubit 4 Fluorometer, Thermo Fisher Scientific, US) (Fig. 5). To demonstrate the performance of our method, we spiked saliva and human serum samples with different concentrations of inactivated SARS-CoV-2 for Np detection. In one single-blind test, 30 virus-spiked and 30 virus-unspiked saliva samples were detected with a Qubit 4 Fluorometer, and all positive samples were detected ( $P<0.001$; Fig. 4b, Figs. S13-S15). In another single-blind test, our method correctly distinguished the 30 positive and 30 negative samples in spiked human serum $(P<0.001$; Fig. 4b). These results indicated that CaT-Smelor-COVID.v3 was ultrasensitive and stable for SARS-CoV-2 detection. 
a

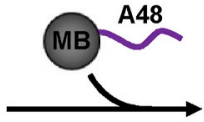

Immobilization

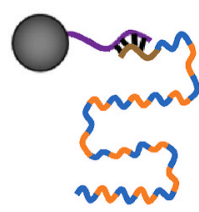

Immobilized

HyDNA10

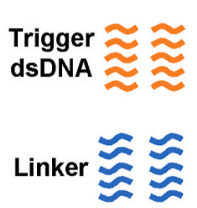

b
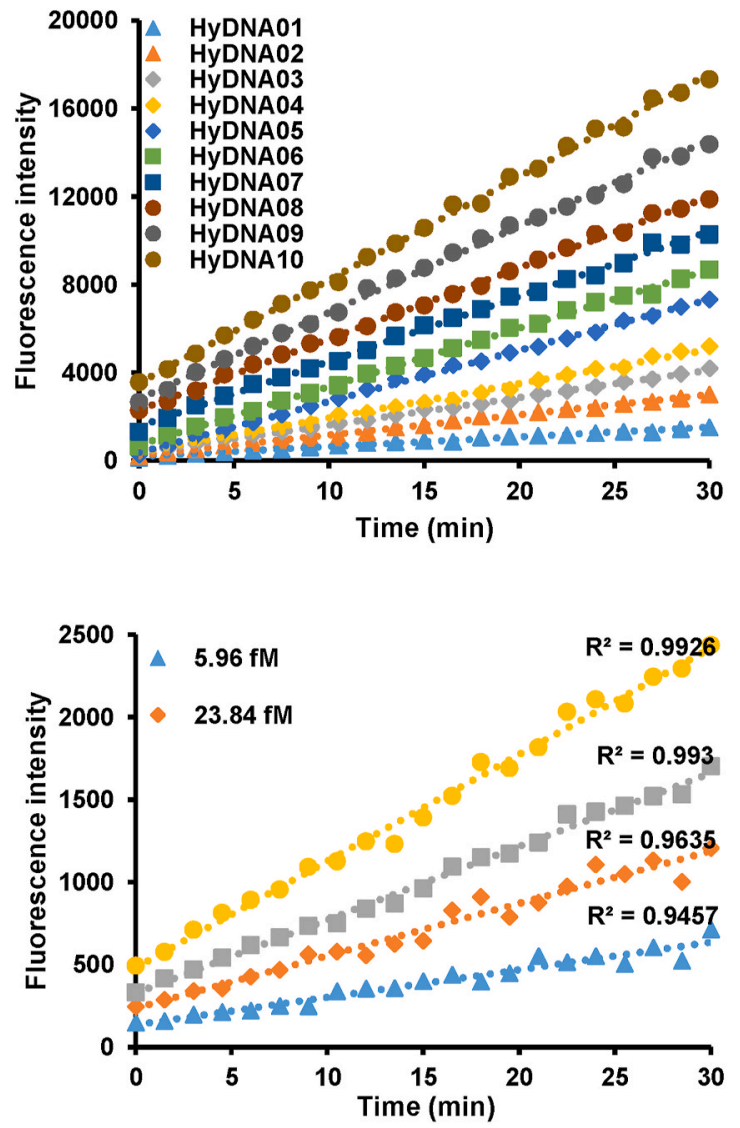

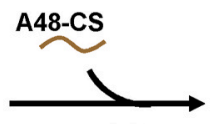

DNA

synthesis

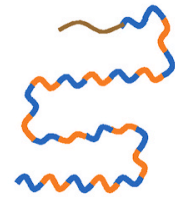

HyDNA10

(Multiple trigger

dsDNA in

tandem)

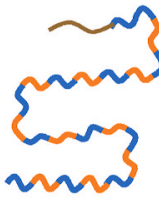

Free HyDNA10

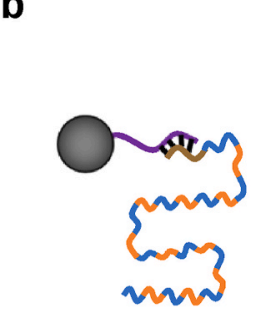

C

\section{f}

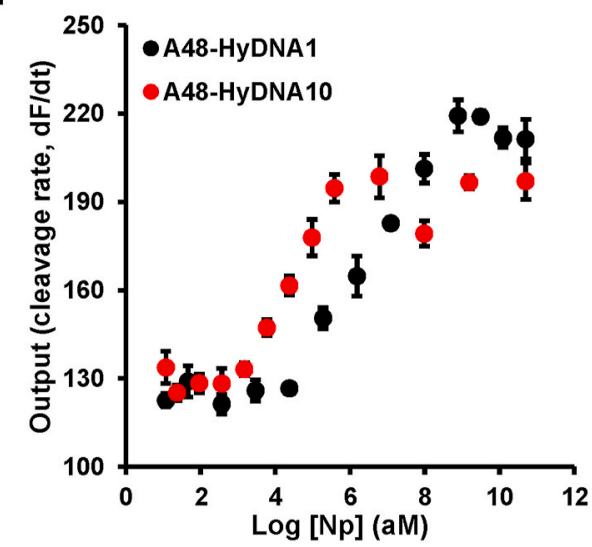

Fig. 2. Detection of Np with the A48-10 based CaT-Smelor-Covid.v2 platform. (a) Constructing schematic diagram of cluster of multiple triggering dsDNAs in tandem. HyDNA10: HyDNA containing ten Cas12a trigger dsDNAs. (b) Sensing schematic diagram of the CaT-Smelor-Covid.v2 platform. (c) Plot of the fluorescence level against the HyDNAs. (d) The slopes of the fluorescence signal are proportional to the copy numbers of Cas12a trigger dsDNA. (e) Readout of the fluorescence signal by the CaT-Smelor-Covid.v2 platform in response to Np. The background signal (control) has been subtracted from the values displayed in the graph. (f) Relationship between the slope and Np concentration output with the CaT-Smelor-Covid.v2 platform and the CaT-Smelor-Covid.v1 platform. In c and e, data are the means and SD of three independent replicates.

\subsection{Perspectives on the state-of-the-art, signal amplifiable CRISPR/ Cas12a aptamer-mediated protein detection platform}

Comparison our new Cas12a-aptamer-based protein detection platform with other previously reported SARS-CoV-2 virus detection methods indicated that CaT-Smelor-Covid.v3 was more sensitive and accurate in rapid antigen detection. The results of our method are comparable to those of the gold-standard RT-qPCR assays and other CRISPR-based nucleic acid detection approaches [11,13] (Table 1). In addition, our rational optimization procedures applied to the 
a

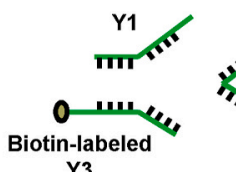

Y3
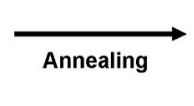

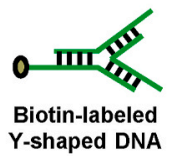

Y-shaped DNA
TIIIII Adapter1-A48

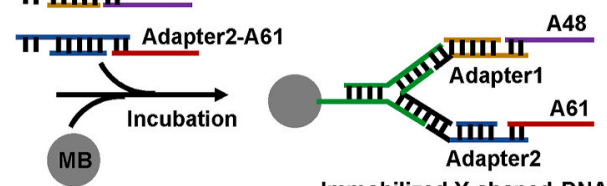

Immobilized Y-shaped DNA

(with adapter and aptamer)

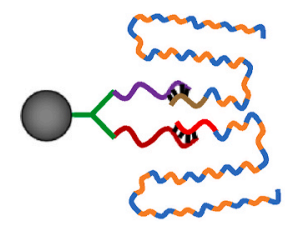

Immobilized A48-HyDNA10 and A61-HyDNA10

A48-HyDNA10

Incubation

b
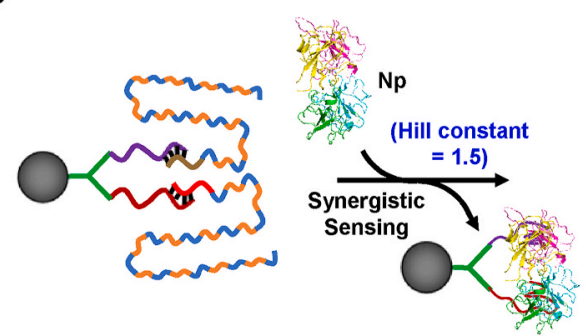

C

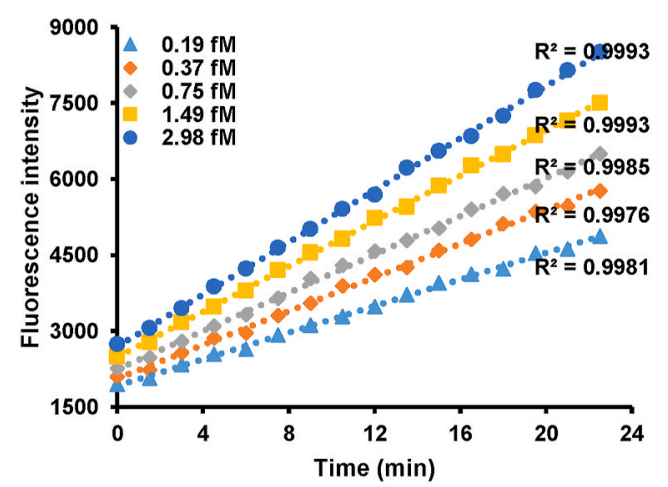
A61-CS

III

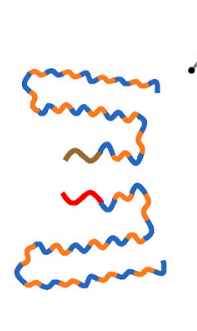

d

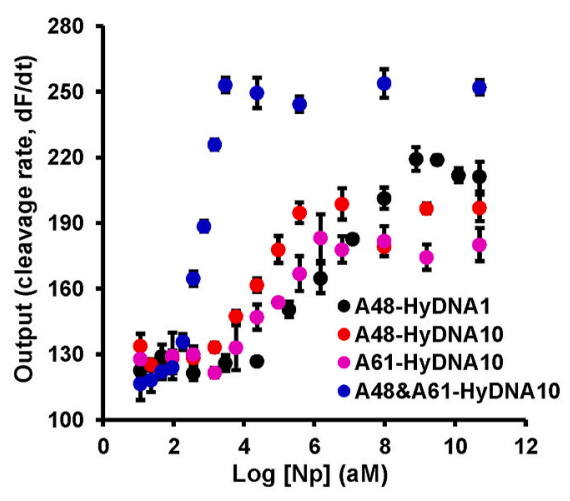

Fig. 3. Detection of Np with the CaT-Smelor-Covid.v3 platform. (a) Design and construction of the dual aptamer synergetic module. Y1, Y2 and biotin labeled Y3 oligonucleotides were complementary to one another. A mixture of the same molar amount of these oligonucleotide strands formed a biotin-labeled Y-shaped DNA. The MB-Y-A48 + A61 complex was constructed through mixture of Y-DNA, MB, Adapter1-A48, and Adapter2-A61. Assembly of the Y-A48 + A61-HyDNA10 complex was accomplished through mixture of MB-Y-A48 + A61, com10-HyDNA10, and com11-HyDNA10. (b) Sensing schematic diagram of the CaT-Smelor-Covid.v3 platform. (c) Readout of the fluorescence signal by the CaT-Smelor-Covid.v3 platform in response to Np. The background signal (control) has been subtracted from the values displayed in the graph. (d) Relationship between the slope and Np concentration output of the biosensing platforms. The readout of the fluorescence signal by the A61-10 based CaT-Smelor-Covid.v2 platform is shown in Fig. S10. The data are the means and SD of three independent replicates.

CaT-Smelor platform successfully boosted the signal about 200 -fold. Given its rational and modular configuration, our system after optimization should be widely applicable in many protein detection-based applications.

\section{Discussion}

In parallel to the nucleic acid detection systems DETECTR, SHEROLOCK, and STOPCovid, the combination of CRISPR/Cas12a and aptamers represents a new detection paradigm for antigen detection [34-37]. Coupling with aptamers dramatically broadens the detection scope of CRISPR/Cas12a [15,22]. However, how to stably amplify the signals sensed by aptamers and then translate the amplified signal into Cas12a cleavage activity has been an unresolved problem. Because of the lack of stable amplification of the signal sensed by the aptamer, this new detection paradigm has been unable to match the sensitivity of DETECTR and SHEROLOCK. Similarly, the sensitivity of Np antigen detection through the combination of CRISPR/Cas12a and aptamer in this study (LOD of $32 \mathrm{fM}$; ca. 193 copies/ $\mu \mathrm{L}$; Fig. 1, Fig. S4) was much lower than the nucleic acid detection systems DETECTR, SHEROLOCK, and STOPCovid, although the performance of CaT-Smelor-Covid.v1 was comparable to those of the previously reported immunology-based methods (186 viral copies/ $\mu \mathrm{L}$ ) [33], Therefore, novel strategies were needed to dramatically enhance the sensitivity of this new detection paradigm. Furthermore, our results represent the general challenges of low sensing efficiency and detection sensitivity in the detection of target analytes through a combination of CRISPR/Cas12a and aptamers. Therefore, the main goal of this study was to address these problems and to provide a general solution for this detection paradigm.

In this study, multiple CRISPR/Cas12a trigger dsDNAs (up to 10 copies) were designed and constructed in tandem in a single DNA fragment (Fig. 2). The use of these trigger dsDNAs significantly amplified the output fluorophore signal. Of note, when the trigger-dsDNA copy number was increased from one (HyDNA) to 10 (HyDNA10), the 
a

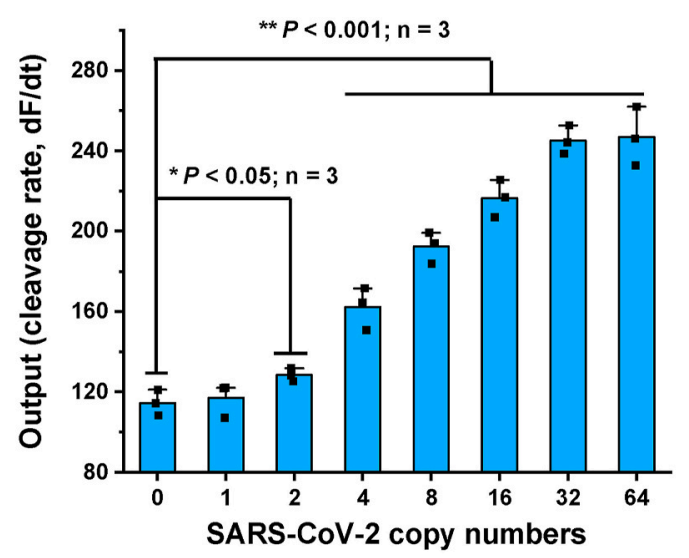

b

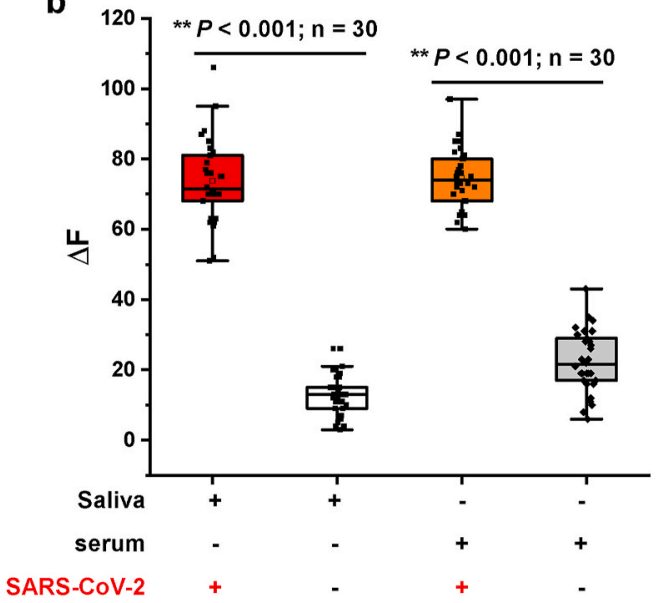

Fig. 4. Detection of clinical inactivated SARS-CoV-2 virus. (a) Detection of clinical inactivated SARS-CoV-2 virus detected by CaT-Smelor-Covid.v3 platform. Data and SD are the means of three independent replicates. (b) Single blind test results of human saliva or serum samples spiked with or without inactivated SARS-CoV-2. $\Delta$ FI: fluorescence intensity increased in $1 \mathrm{~min}$. The test results showed that the correct detection rate was $100 \%$.
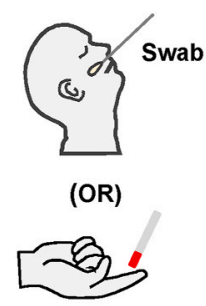

Serum / saliva

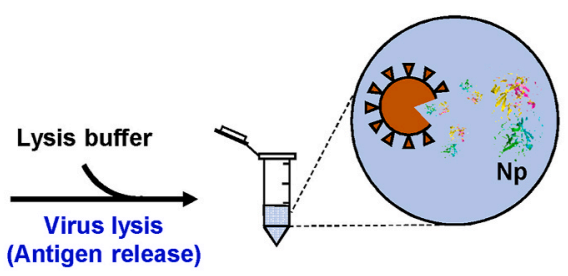

(Antigen release)
$(15 \sim 20 \mathrm{~min})$

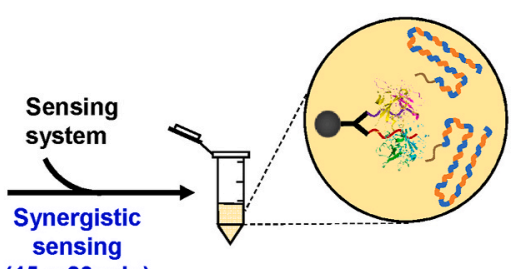

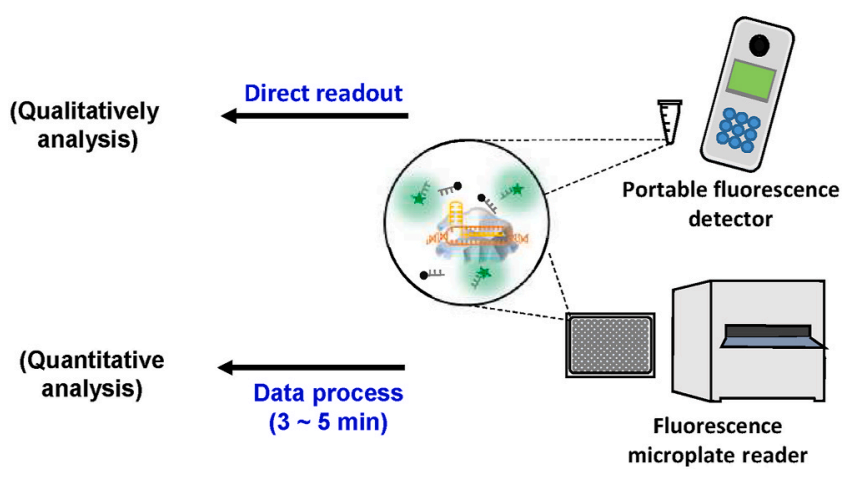

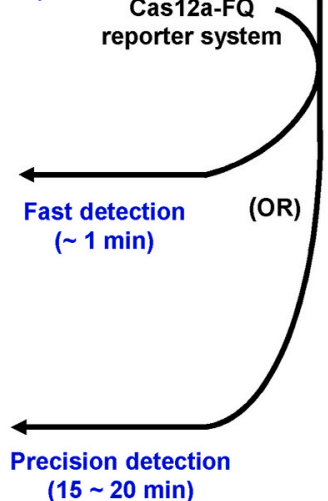

$(15 \sim 20 \mathrm{~min})$

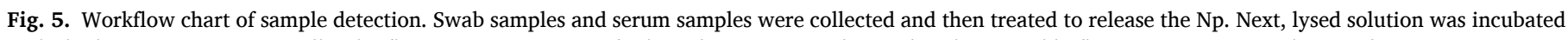
with the biosensor system. Finally, the fluorescence intensity of released HyDNA was detected with a portable fluorometer or microplate reader.

detection sensitivity also linearly increased approximately 10-fold (from $32 \mathrm{fM}$ to $3.48 \mathrm{fM})$. Warranting the stabilized output signal and maximization of the $\mathrm{S} / \mathrm{N}$, we designed both the crRNA and trigger dsDNA sequences on the basis of the phage genome-derived prediction site [38], which ensure optimal and robust performance of our CRISPR/Cas12a-based signal output system. In addition, the intersequence region between each trigger dsDNA was optimized. On the basis of our results, we believe that this well-characterized multiple trigger dsDNA tandem element could be used as a general tool in other CRISPR/Cas12a and aptamer-based detection systems, such as CaT-Smelor [38], in applications beyond Np detection.

For most aptamers, the affinity toward the corresponding target is usually at the nanomolar level (i.e., dissociation constant, $K_{D}$ ), and further increasing the binding affinity by systematic evolution of ligands by exponential enrichment (SELEX) may be difficult [39]. However, the $K_{D}$ of the aptamer directly determines the sensing efficiency and consequently affects the detection sensitivity. Therefore, innovating the aptamer-based sensing module for a more sensitive response to antigen, independently of the affinity between aptamer and antigen, should be of general interest for all aptamer-based detection systems. In this study, we used a Y-shaped DNA and two aptamers, A48 and A61, recognizing different epitopes of the antigen, which were designed to sense the Np (Fig. 3). A significant synergistic sensing effect was observed, in which the binding of A48 to Np facilitated the binding of A61 and Np, and vice versa. According the response curve, the Hill coefficient reached 1.52, thus indicating a positive synergistic effect between A48 and A61 [40]. By combining the multiple trigger dsDNA and synergistic sensing strategies, our system achieved an LOD for Np detection of $0.17 \mathrm{fM}$ (Fig. S10). Therefore, our dual aptamer synergistic sensing strategy, independently of binding affinity, not only increased the affinity limit of aptamers but also further improved the detection sensitivity (from 3.48 $\mathrm{fM}$ to $0.17 \mathrm{fM}$ ). Moreover, the synergistic effects could be applied to the detection of any other antigen with two aptamers that recognize different epitopes of the antigen. 
Table 1

Comparison of reliable SARS-CoV-2 detecting methods.

\begin{tabular}{|c|c|c|c|c|c|c|}
\hline Method & Target & LOD & $\mathrm{V}$ & $\mathrm{TC}(\mathrm{min})$ & $\operatorname{Bir}(\mathrm{Y} / \mathrm{N})$ & Ref. \\
\hline RT-LAMP & RNA & 50 copies $/ \mu \mathrm{L}$ & $2 \mu \mathrm{L}$ & 30 & $\mathrm{Y}$ & Ganguli A et al. [30] \\
\hline RT-qPCR & RNA & 100 copies $/ \mu \mathrm{L}$ & $5 \mu \mathrm{L}$ & 120 & $\mathrm{Y}$ & Vogels CBF et al. [31] \\
\hline Cas13a based detection & RNA & $\sim 100$ copies $/ \mu \mathrm{L}$ & $\sim 0.3 \mu \mathrm{L}$ & 30 & $\mathrm{~N}$ & Fozouni P et al. [17] \\
\hline DETECTR & RNA & 10 copies $/ \mu \mathrm{L}$ & $2 \mu \mathrm{L}$ & $30-40$ & $\mathrm{~N}$ & Broughton JP et al. [11] \\
\hline AIOD-CRISPR & RNA & $\sim 5$ copies $/ \mu \mathrm{L}$ & $1 \mu \mathrm{L}$ & $>40$ & $\mathrm{~N}$ & Ding $X$ et al. [14] \\
\hline STOPCovid & RNA & 33 copies $/ \mathrm{mL}$ & $50 \mu \mathrm{L}$ & $>125$ & $\mathrm{~N}$ & Joung J et al. [13] \\
\hline Electrochemical biosensor & RNA & 200 copies/mL & $10 \mu \mathrm{L}$ & $>180$ & $\mathrm{~N}$ & Zhao H et al. [32] \\
\hline Microfluidic immunoassays & Antibody & N. D & $10 \mu \mathrm{L}$ & 15 & $\mathrm{Y}$ & Lin Q et al. [33] \\
\hline MNPs biosensor & Antigen & $5.06 \times 10^{7}$ copies $/ \mu \mathrm{L}$ & $70 \mu \mathrm{L}$ & $\sim 10$ & $\mathrm{Y}$ & Zhong J et al. [26] \\
\hline LFIA & Antigen & 186 copies $/ \mu \mathrm{L}$ & $<5$ ng protein & $>20$ & $\mathrm{~N}$ & Lee JH et al. [3] \\
\hline CaT-Smelor-Covid.v3 & Antigen & 2 copies $/ \mu \mathrm{L}$ & $1 \mu \mathrm{L}$ & $\sim 20$ & $\mathrm{~N}$ & This study \\
\hline
\end{tabular}

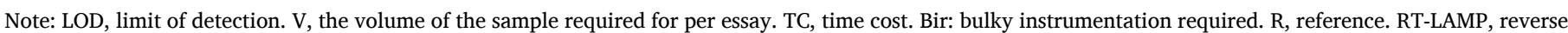

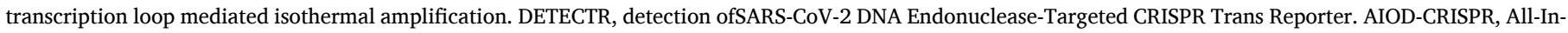

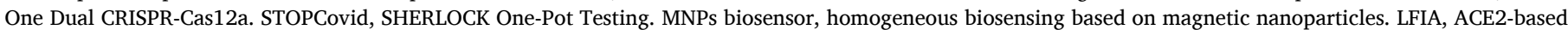
lateral flow immunoassay. N.D, not determined.

The output of the ssDNA cleavage activity of Cas12a can detected through many convenient methods, e.g., lateral-flow [41,42]. Here, we used a commercially available portable fluorescence detector to demonstrate the merits and potential of our method in clinical diagnosis. Saliva and serum spiked with different concentrations of inactivated SARS-CoV-2 were used to mimic clinical samples, and the positive detection rate reached $100 \%$ (Fig. $4 \mathrm{~b}$ ). Despite the limitations of the experimental conditions, and the absence of clinical trials, we believe that our results confirmed the practicability of our detection approach. In addition, compared with the existing antigen detection methods, our method had significant advantages in that the sensitivity was greatly improved, and the testing is fast in time-spent and low in cost (Table 1). The abovementioned advantages of CRISPR/Cas-based detection systems also extended to any antigen detection with our strategy.

Consequently, by focusing on the issues of signal amplification and sensing efficiency in the CRISPR/Cas12a and aptamer-based detection paradigm, we not only developed a PCR-free multiple trigger dsDNA tandem-based signal amplification strategy for high performance CRISPR/Cas-based detection but also designed a dual aptamer synergistic sensing strategy for highly efficient sensing. Integrating these two aspects, we achieved the first reported ultrasensitive, fast, stable, highperformance antigen detection using a CRISPR/Cas12a and aptamerbased detection paradigm. Given that aptamers recognizing different fragments of variable proteins (for example, multiple epitopes of antigens) can be generated by SELEX, we believe that the innovative strategy used in this study may mark the beginning of a wide range of applications in the development of detection methods for antigens and other target molecules.

\section{Declaration of competing interest}

The authors indicate that they have no conflict of interest.

\section{CRediT authorship contribution statement}

Xiangxiang Zhao: Methodology, Investigation, Data curation, Visualization, Writing - original draft, Writing - review \& editing. Zhengduo Wang: Investigation, Writing - original draft. Bowen Yang: Investigation, Visualization, Writing - original draft. Zilong Li: Resources. Yaojun Tong: Writing - review \& editing. Yuhai Bi: Provided inactivated virus samples. Zhenghong Li: Resources. Xuekui Xia: Resources. Xiangyin Chen: Resources. Lixin Zhang: Funding acquisition, Writing - review \& editing. Weishan Wang: Funding acquisition, Writing - original draft. Gao-Yi Tan: Conceptualization, Supervision, Data curation, Visualization, Funding acquisition, Writing - original draft, Writing - review \& editing.

\section{Acknowledgements}

This work was supported by the National Key R\&D program of China (2020YFA0907800), the National Natural Science Foundation of China (31922002, 31720103901, 31772242 and 31870040), the 111 Project (B18022), the Fundamental Research Funds for the Central Universities [22221818014], the Youth Innovation Promotion Association CAS (Y202027) to W.W and the Open Project Funding of the State Key Laboratory of Bioreactor Engineering.

\section{Appendix A. Supplementary data}

Supplementary data to this article can be found online at https://doi. org/10.1016/j.synbio.2021.09.007.

\section{References}

[1] Wang C, Horby PW, Hayden FG, Gao GF. A novel coronavirus outbreak of global health concern. Lancet 2020;395:470-3. https://doi.org/10.1016/S0140-6736(20) 30185-9. 10223.

[2] Barr JN, Fearns R. In: Kovalchuk Igor, Kovalchuk Olga, editors. Chapter 2 - genetic in stability of RNA viruses. Boston: Academic Press; 2016. p. 21-35. https://doi. org/10.1016/B978-0-12-803309-8.00002-1.

[3] Lee J-H, Choi M, Jung Y, et al. A novel rapid detection for SARS-CoV-2 spike 1 antigens using human angiotensin converting enzyme 2 (ACE2). Biosens Bioelectron 2021;171:112715. https://doi.org/10.1016/j.bios.2020.112715.

[4] Serrano MM, Rodríguez DN, Palop NT, et al. Comparison of commercial lateral flow immunoassays and ELISA for SARS-CoV-2 antibody detection. J Clin Virol 2020;129:104529. https://doi.org/10.1016/j.jcv.2020.104529.

[5] Van Elslande J, Decru B, Jonckheere S, et al. Antibody response against SARS-CoV2 spike protein and nucleoprotein evaluated by four automated immunoassays and three elisas. Clin Microbiol Infect 2020;26:1557. https://doi.org/10.1016/j. cmi.2020.07.038. e1551-1557.e1557.

[6] Boum Y, Fai KN, Nicolay B, et al. Performance and operational feasibility of antigen and antibody rapid diagnostic tests for COVID-19 in symptomatic and asymptomatic patients in Cameroon: a clinical, prospective, diagnostic accuracy study. Lancet Infect Dis 2021;21(8):1089-96. https://doi.org/10.1016/S14733099(21)00132-8.

[7] Liu D, Ju C, Han C, et al. Nanozyme chemiluminescence paper test for rapid and sensitive detection of SARS-CoV-2 antigen. Biosens Bioelectron 2021;173:112817. https://doi.org/10.1016/j.bios.2020.112817.

[8] Sitjar J, Liao J-D, Lee H, et al. Challenges of SERS technology as a non-nucleic acid or -antigen detection method for SARS-CoV-2 virus and its variants. Biosens Bioelectron 2021;181:113153. https://doi.org/10.1016/j.bios.2021.113153.

[9] Pinals RL, Ledesma F, Yang D, et al. Rapid SARS-CoV-2 spike protein detection by carbon nanotube-based near-infrared nanosensors. Nano Lett 2021;21:2272-80. https://doi.org/10.1021/acs.nanolett.1c00118.

[10] Notomi T, Okayama H, Masubuchi H, et al. Loop-mediated isothermal amplification of DNA. Nucleic Acids Res 2000;28. https://doi.org/10.1093/nar/ 28.12.e63. e63-e63.

[11] Broughton JP, Deng X, Yu G, et al. CRISPR-Cas12-based detection of SARS-CoV-2. Nat Biotechnol 2020;38:870-4. https://doi.org/10.1038/s41587-020-0513-4.

[12] Gootenberg JS, Abudayyeh OO, Lee JW, et al. Nucleic acid detection with CRISPRCas13a/C2c2. Science 2017;356:438. https://doi.org/10.1126/science.aam9321.

[13] Joung J, Ladha A, Saito M, et al. Detection of SARS-CoV-2 with SHERLOCK one-pot testing. N Engl J Med 2020;383:1492-4. https://doi.org/10.1056/NEJMc2026172. 
[14] Ding X, Yin K, Li Z, et al. Ultrasensitive and visual detection of SARS-CoV-2 using all-in-one dual CRISPR-Cas12a assay. Nat Commun 2020;11:4711. https://doi.org/ 10.1038/s41467-020-18575-6.

[15] Zhao X, Li S, Liu G, et al. A versatile biosensing platform coupling CRISPR-Cas12a and aptamers for detection of diverse analytes. Sci Bull 2021;66:69-77. https:// doi.org/10.1016/j.scib.2020.09.004.

[16] Liang M, Li Z, Wang W, et al. A CRISPR-Cas12a-derived biosensing platform for the highly sensitive detection of diverse small molecules. Nat Commun 2019;10:3672. https://doi.org/10.1038/s41467-019-11648-1.

[17] Fozouni P, Son S, Díaz de León Derby M, et al. Amplification-free detection of SARS-CoV-2 with CRISPR-Cas13a and mobile phone microscopy. Cell 2021;184: 323-33. https://doi.org/10.1016/j.cell.2020.12.001. e329.

[18] Esbin MN, Whitney ON, Chong S, et al. Overcoming the bottleneck to widespread testing: a rapid review of nucleic acid testing approaches for COVID-19 detection. https://doi.org/10.1261/rna.076232.120; 2020. 26, 771-783.

[19] Nouri R, Tang Z, Dong M, et al. CRISPR-based detection of SARS-CoV-2: a review from sample to result. Biosens Bioelectron 2021;178:113012. https://doi.org/ 10.1016/j.bios.2021.113012.

[20] Hermann T, Patel DJ. Adaptive recognition by nucleic acid aptamers. Science 2000;287:820. https://doi.org/10.1126/science.287.5454.820.

[21] Sun H, Zu Y. A highlight of recent advances in aptamer technology and its application. Molecules 2015;20(7):11959-80. https://doi.org/10.3390/ molecules200711959.

[22] Xiong Y, Zhang J, Yang Z, et al. Functional DNA regulated CRISPR-Cas12a sensors for point-of-care diagnostics of non-nucleic-acid targets. J Am Chem Soc 2020;142: 207-13. https://doi.org/10.1021/jacs.9b09211.

[23] Isho B, Abe KT, Zuo M, et al. Persistence of serum and saliva antibody responses to SARS-CoV-2 spike antigens in COVID-19 patients. Sci Immunol 2020;5. https:// doi.org/10.1126/sciimmunol.abe5511. eabe5511.

[24] Xu H, Xia A, Wang D, et al. An ultraportable and versatile point-of-care DNA testing platform. Sci Adv 2020;6:eaaz7445. https://doi.org/10.1126/sciadv.aaz7445.

[25] Li Y, Tseng YD, Kwon SY, et al. Controlled assembly of dendrimer-like DNA. Nat Mater 2004;3:38-42. https://doi.org/10.1038/nmat1045.

[26] Zhong J, Rosch EL, Viereck T, et al. Toward rapid and sensitive detection of SARSCoV-2 with functionalized magnetic nanoparticles. ACS Sens 2021;6:976-84. https://doi.org/10.1021/acssensors.0c02160.

[27] Zhang L, Fang X, Liu X, et al. Discovery of sandwich type COVID-19 nucleocapsid protein DNA aptamers. Chem Commun 2020;56:10235-8. https://doi.org/ 10.1039/d0cc03993d.

[28] Yamano T, Nishimasu H, Zetsche B, et al. Crystal structure of cpf1 in complex with guide RNA and target DNA. Cell 2016;165:949-62. https://doi.org/10.1016/j. cell.2016.04.003.
[29] Yang J, Niu P, Chen L, et al. Genetic tracing of HCoV-19 for the re-emerging outbreak of COVID-19 in beijing, China. Protein Cell 2021;12:4-6. https://doi.org/ 10.1007/s13238-020-00772-0.

[30] Ganguli A, Mostafa A, Berger J, et al. Rapid isothermal amplification and portable detection system for SARS-CoV-2. Proc Natl Acad Sci U S A 2020;117:22727-35. https://doi.org/10.1073/pnas.2014739117.

[31] Vogels CBF, Brito AF, Wyllie AL, et al. Analytical sensitivity and efficiency comparisons of SARS-CoV-2 RT-qPCR primer-probe sets. Nat Microbiol 2020;5: 1299-305. https://doi.org/10.1038/s41564-020-0761-6.

[32] Zhao H, Liu F, Xie W, et al. Ultrasensitive supersandwich-type electrochemical sensor for SARS-CoV-2 from the infected COVID-19 patients using a smartphone. Sensor Actuator B Chem 2021;327:128899. https://doi.org/10.1016/j. snb.2020.128899.

[33] Lin Q, Wen D, Wu J, et al. Microfluidic immunoassays for sensitive and simultaneous detection of IgG/IgM/Antigen of SARS-CoV-2 within 15 min. Anal Chem 2020;92:9454-8. https://doi.org/10.1021/acs.analchem.0c01635.

[34] Li H, Xing S, Xu J, et al. Aptamer-based CRISPR/Cas12a assay for the ultrasensitive detection of extracellular vesicle proteins 2021;221:121670. https://doi.org/ 10.1016/j.talanta.2020.121670.

[35] Li H, Li M, Yang Y, et al. Aptamer-linked CRISPR/Cas12a-based immunoassay 2021;93:3209-16. https://doi.org/10.1021/acs.analchem.0c04687.

[36] Niu C, Wang C, Li F, et al. Aptamer assisted CRISPR-Cas12a strategy for small molecule diagnostics. 2021. p. 113196. https://doi.org/10.1016/j. bios.2021.113196.

[37] Qiao B, Xu J, Yin W, et al. "Aptamer-Locker" DNA coupling with CRISPR/Cas12aguided biosensing for high-efficiency melamine analysis, vol. 113233; 2021. https://doi.org/10.1016/j.bios.2021.113233.

[38] Chen JS, Ma E, Harrington LB, et al. CRISPR-Cas12a target binding unleashes indiscriminate single-stranded dnase activity. Science 2018;360:436. https://doi. org/10.1126/science.aar6245.

[39] Darmostuk M, Rimpelova S, Gbelcova H, et al. Current approaches in selex: an update to aptamer selection technology. Biotechnol Adv 2015;33:1141-61. https://doi.org/10.1016/j.biotechadv.2015.02.008.

[40] Simon AJ, Vallée-Bélisle A, Ricci F, et al. Using the population-shift mechanism to rationally introduce "Hill-type" cooperativity into a normally non-cooperative receptor. Angew Chem Int Ed 2014;53:9471-5. https://doi.org/10.1002/ anie. 201403777.

[41] Gootenberg JS, Abudayyeh OO, Kellner MJ, et al. Multiplexed and portable nucleic acid detection platform with Cas13, Cas12a, and Csm6. Science 2018;360:439. https://doi.org/10.1126/science.aaq0179.

[42] Ali Z, Aman R, Mahas A, et al. Iscan: an RT-LAMP-coupled CRIPSR-Cas12 module for rapid, sensitive detection of SARS-CoV-2. Virus Res 2020;288:198129. https:// doi.org/10.1016/j.virusres.2020.198129. 